\title{
Psychological Treatment for Headache: A Pilot Study on the Efficacy of Joint Psychoeducational Group and Relaxation Training
}

Elisa Andrighi ${ }^{1}$, Federica Galli ${ }^{*}$, Nicoletta Massetto ${ }^{1}$, Irene Strada ${ }^{1}$, Serena Magno ${ }^{1}$, Anna Dodi $^{1}$ and Elena Vegni ${ }^{1,2}$

${ }^{1}$ ASST S. Paolo and S. Carlo Hospital, Via A. Di Rudini, 8-20142, Milan, Italy

${ }^{2}$ Department of Health Sciences, University of Milan, Milan, Italy

*Corresponding author: Federica Galli, Department of Health Sciences, University of Milan, Milan, Italy, Tel: 0039-02-50323129; E-mail: federica.galli1@unimi.it

Received date: April 18, 2016; Accepted date: June 17, 2016; Published date: June 24, 2016

Copyright: ( 2016 Andrighi, et al. This is an open-access article distributed under the terms of the Creative Commons Attribution License, which permits unrestricted use, distribution, and reproduction in any medium, provided the original author and source are credited.

\begin{abstract}
Headache is a severe and disabling chronic condition. Literature shows that psycho-educational interventions may be useful for several chronic pain disorders. We tested an integrated psycho-educational and relaxation intervention addressed to patients suffering from headache.

The outcome indicators are a direct measure of headache (headache days per month) and two indirect measures (disability due to headache and medications used). At the end of the intervention patients also filled in a satisfaction questionnaire. Forty-seven subjects participated to the study. The treatment significantly reduced frequency of headache attacks (Wilcoxon $p<0.01$ ) and disability caused by headache (Wilcoxon $p<0.001$ ). There was also a sensible reduction in medication use. Almost all patients judged the intervention positively. An integrated approach may be very useful to ameliorate the burden of headache, to discontinue medication and to improve quality of life.
\end{abstract}

Keywords: Migraine; Chronic headache; Non-drug therapy; Autogenic training; Psychoeducational intervention

\section{Introduction}

Headache is a severe and disabling chronic disease. The Global Burden of Disease Study 2010 identified migraine as the "seventh disabler" because of its high prevalence and impact on life $[1,2]$. Migraine is also associated with high costs on the individual as well as on the society $[3,4]$. The worldwide prevalence of primary headache in adults is $46 \%$, but rises to $79.6 \%$ if we consider only the European population (71.1\% of men and $86 \%$ of women) [5]. An Italian epidemiological study [6] identified a headache prevalence of $69.1 \%$ (75.8\% for women and $60.6 \%$ for men; M:F ratio=1:2).

Furthermore, headache is a complex neurological condition that affects all aspects of the individual's life and is considered the result of interaction between biological, psychological and environmental factors. The role of psychopathological factors in headache disorders has been widely studied in the last twenty years [7] and according to several studies has a negative burden on the prognosis across different headache sub-types $[8,9]$. Some authors suggest that it is a biobehavioral disorder, the result of the interplay between cortical hypersensitivity and a social learning process [10-12]. In addition, it has been also highlighted a correlation between stress intensity and headache frequency [13].

The best approach to deal with headache, as well as with other kind of chronic pain where it does exist an important interplay of biological and psychological factors seems to be a combination of pharmacological and non-pharmacological treatments [14-17].

Literature shows that non-pharmacological interventions, including cognitive stress management, behavioral training, relaxation techniques (associated or not with biofeedback) reduce frequency and/or intensity of headache attacks and improve the quality of life $[18,19]$.

The term psychoeducation means interventions that aim to increase patients' emotional ability to cope with their disease and to improve their knowledge's about disease and therapy. The effectiveness of psychoeducational interventions in treating several chronic diseases is known [20-23]. World Health Organization (WHO) affirms that trained health workers should provide such an education, in order to give information to the patients about treatment management, helping them to avoid complication and engaging patients in their own care path to achieve the best possible quality of life [23-25].

Wallasch and colleagues [26] evaluated a multidisciplinary intervention specific for headache that included patient education, cognitive behavioral therapy and relaxation practices, reporting an attacks frequency reduction of $62.7 \%$, a disability degree reduction of 47.4 and an analgesic use reduction of $74.5 \%$. Even Kindelan-Calvo and colleagues, in their review of 2014 [16] showed the effectiveness of some psychoeducational interventions in reducing frequency of headache and disability associated with it, with an improved quality of life.

Aim of this study was evaluating the effectiveness of an integrated psycho-educational and relaxation training intervention in order to prevent the burden of headache.

\section{Materials and Methods}

Forty-seven subjects were enrolled in the study (age range: 23-71; m.a.46.1; +8.3$) ; 85 \%$ were women $(n=39)$ and about $40 \%$ were clerical workers (Table 1).

With regard to diagnoses, the sample was composed as shown in Table 1. 


\begin{tabular}{|l|c|}
\hline \multicolumn{1}{|c|}{ Subjects $-\mathbf{n}$} & 47 \\
\hline Mean age - Age range & $42[23-71]$ \\
\hline Male sex $-\mathbf{n}(\%)$ & $8(17)$ \\
\hline Employment $-\mathbf{n}(\%)$ & $19(40)$ \\
\hline Employee & $7(15)$ \\
\hline Teacher & $5(11)$ \\
\hline Student & $5(11)$ \\
\hline Pensioner & $3(6)$ \\
\hline Nurse & $1(2)$ \\
\hline Unemployed & $7(15)$ \\
\hline Other & \\
\hline Diagnosis - $\mathbf{n}$ (\%) & $18(38.3)$ \\
\hline Migraine (with or without aura) & $12(25.55)$ \\
\hline Chronic Migraine & $5(10.6)$ \\
\hline Episodic Tension type headache & $12(25.55)$ \\
\hline Migraine and tension-type headache & \\
\hline
\end{tabular}

social relationships, doubts and curiosities about headache (two "cognitive" meetings, one of them with the neurologist's participation), group closure and observations about the experience into the group of each member.

The second phase of each meeting aimed at learning relaxation techniques (in particular, heaviness, face and shoulders exercises of Autogenic Training).

The intervention was designed in 8 weekly group-meetings, lasting each one 90 minutes. Each of the 8 meetings was divided into two phases: an initial one focused on emotional sharing through active methodologies of psychodrama or exploration/increasing of knowledge about headache and a second one aimed at learning relaxation techniques (Autogenic Training).

\section{Outcome Indicators}

According to Andrasik et al. [28], we considered as outcome indicators both direct measures of headache (headache days per month) and indirect measures (disability due to headache and medications used). In particular, we examined:

- Attacks frequency, expressed in days per month, collected from the "headache diary" filled in by each patient

- Headache related disability in social life or work, evaluated by the mean of MIDAS questionnaire (Migraine Disability Assessment Score - the questionnaire allows to identify a disability value ranging from 0 (no disability) to 4 (maximum degree of disability))

Table 1: Sample description.

\section{Inclusion Criteria}

- Age $>18$ years

- Absence of severe psychiatric disorder (s) (e.g. psychosis)

- Diagnosis of primary headache according to the ICHD-III- $\beta$ criteria [27]

- Willingness to have a psychological support

- Suitability to the techniques of Autogenic Training (e.g. ability to use imagination and willingness to practice relaxation exercises at home).

\section{Psycho-Educational and Relaxation Intervention}

The neurologists (NM, SM) of the Headache Center of the S. Paolo Hospital of Milan selected patients who fell in the first three inclusion criteria. In a second step, psychologists made the final recruitment through individual interviews aimed to assess the suitability of patients to the treatment, by verifying the presence of the other inclusion criteria and had difficulty in stress managing.

The recruitment was carried out at the Clinical Psychology Unit through individual interviews aimed to assess the suitability of patients to the treatment, by verifying the presence of the other inclusion criteria, and to explain the proposed intervention.

The intervention was designed in 8weekly group-meetings, lasting each one 90 minutes, and was conducted by two psychologists with the participation of a neurologist in one of the meetings.

Each of the 8 meetings was composed of two phases. The first one was focused on sharing thoughts, emotions and knowledges about headache. Each meeting dealt with different issues: mutual knowing and establishment of the group, different headache experiences, headache related emotions, personal resources, headache effect on

- Medications use (prophylactic, symptomatic or none)

- Participants' level of satisfaction was detected by an ad hoc questionnaire, filled in by patients at the end of the treatment.

The psychoeducational intervention effectiveness were evaluated comparing the data of frequency, disability and medication use gathered the month before the inception of treatment with those gathered the month after the intervention.

\section{Statistical Analysis}

The data were analyzed using a nonparametric test for paired data (test of Wilcoxon rank sum), comparing those before intervention with those after.

For statistical analysis was used the statistical package STATA 12 (Stata Corporation, College Station, Texas, USA).

\section{Results}

Before the treatment $34 \%$ of the patients $(n=16)$ used symptomatic drugs (common analgesics, triptans, combination medications) and the remaining $66 \%(\mathrm{n}=31)$ took a prophylactic therapy (calcium antagonists, b-blockers, tricyclic antidepressants with or without neuroleptics or antiepileptics).

After the intervention we found a significant improvement (Wilcoxon $\mathrm{p}<0.01)$ : $36 \%$ of the participants $(\mathrm{n}=17)$ reduced medication use, $57.5 \%(n=27)$ maintained the same drug therapy and only $6.5 \%$ $(n=3)$ had a worsening of the situation (moving from symptomatic to prophylactic therapy). As improvement, we considered the change from prophylactic to symptomatic therapy or from symptomatic to no therapy (Table 2). 
Citation: Andrighi E, Galli F, Massetto N, Strada I, Magno S, et al. (2016) Psychological Treatment for Headache: A Pilot Study on the Efficacy of Joint Psychoeducational Group and Relaxation Training. J Neurol Neurophysiol 7: 379. doi:10.4172/2155-9562.1000379

Page 3 of 5

\begin{tabular}{|l|c|c|c|}
\hline & Before & After & p \\
\hline Therapy $\mathbf{n}$ (\%) & & & \\
\hline Symptomatic & $16(34)$ & $23(49)$ & \\
\hline Prophylaptic & $31(66)$ & $18(38)$ & $<0.01$ \\
\hline None & $0(0)$ & $6(13)$ & \\
\hline Attacks frequency $\mathbf{n}$ (\%) & & & \\
\hline$<5$ days per month & $7(14.9)$ & $22(46.8)$ & \\
\hline $5-9$ days per month & $12(25.5)$ & $12(25.5)$ & $<0.01$ \\
\hline 10-15 days per month & $10(21.3)$ & $7(14.9)$ & \\
\hline$>15$ days per month & $18(38.3)$ & $6(12.8)$ & \\
\hline Disability degree & $3[1-4]$ & $1[0-4]$ & $<0.001$ \\
\hline
\end{tabular}

Table 2: Comparison of medication use, attacks frequency and disability before and after the treatment.

Among improved patients, $47 \%(\mathrm{n}=8)$ suffered from migraine (with or without aura), $29.4 \%(n=5)$ from Chronic Migraine, $17.6 \%(n=3)$ migraine and tension-type headache and $6 \%(n=1)$ from episodic tension type headache. Examining all the participants, the improvement pertained to $44.4 \%$ of patients suffering from migraine, to $41.7 \%$ of patients suffering from Chronic Migraine, to $25 \%$ of patients suffering from mixed forms and to $20 \%$ of patient suffering from frequent episodic tension-type headache. Furthermore, greatest benefits were obtained in $94 \%$ of cases by the patients using initially prophylactic therapy $(n=16)$. In particular, $51.6 \%$ of these patients experienced an improvement, in comparison to the $6 \%(n=1)$ of those taking symptomatic therapy. Regarding headache frequency before the intervention, $14.9 \%$ of the subjects had less than 5 days with headache per month, $25.5 \%$ between 5 and 9 days, $21.3 \%$ between 10 and 15 days and the remaining $38.3 \%$ over 15 days. The treatment was effective in reducing the frequency of headache attacks (Wilcoxon $\mathrm{p}<0.01$ ), with an average reduction of 7.02 days with headache per month (Table 2 and Figure 1).

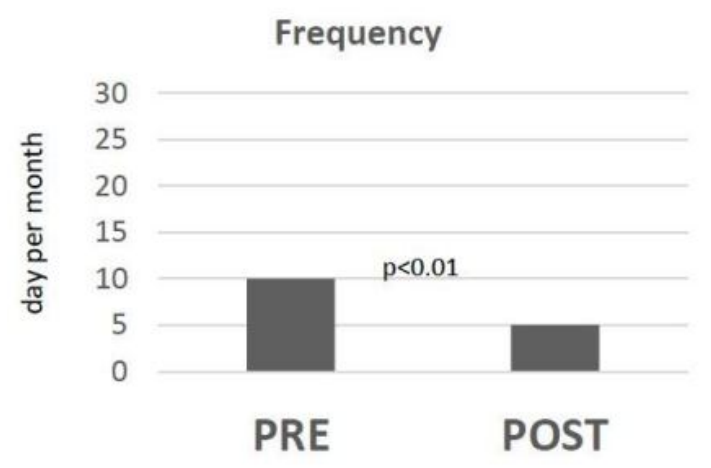

Figure 1: Headache frequency before and after the treatment. (Headache frequency (days per month) median value decreased after the treatment (Wilcoxon $\mathrm{p}<0.01$ ).

Before the intervention headache related disability was on average 2.94 (median=3); the treatment brought a statistically significant reduction $($ mean $=1.64$, median $=1)$ (Wilcoxon $\mathrm{p}<0.001$ ) (Table 2 and Figure 2).

Finally, the overall opinion expressed by the participants was very positive (mean score 4.63 on a 5 -point Likert scale); the intervention was also considered useful (4.31 out of 5), satisfied the participants' expectations (4.13 out of 5) and would be recommended to persons suffering from headache (4.75 out of 5 ). Despite the recommendation of daily exercising, patients performed relaxation exercises on average 1-2 times a week (Figure 3).

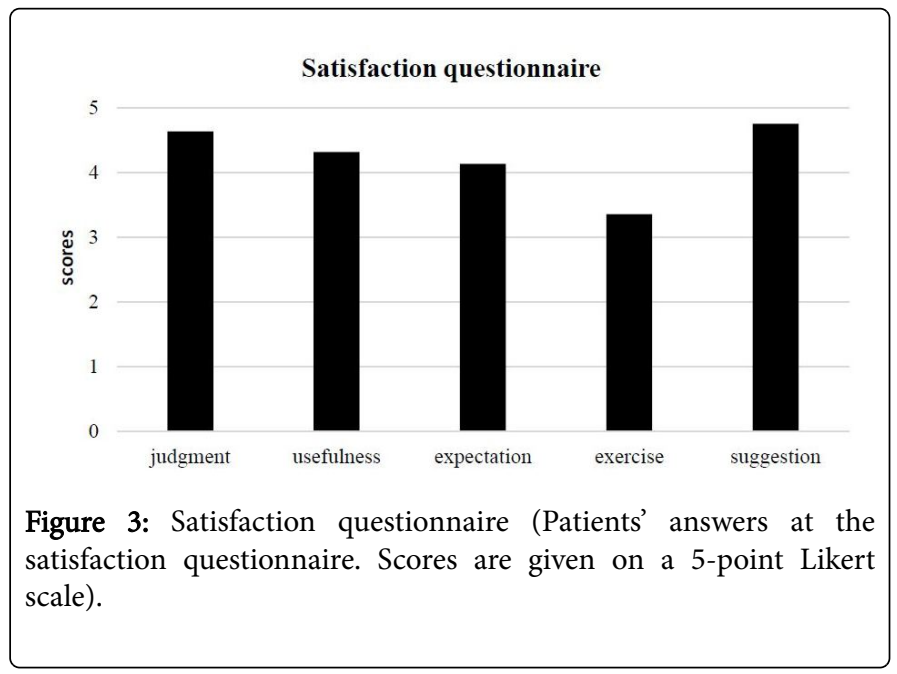

\section{Discussion}

The proposed treatment was effective in reducing the headache frequency of $45.9 \%$ and headache related disability degree of $44.2 \%$. There was also a significant improvement in medication use, with $36 \%$ of participants reporting a reduction, from prophylactic to symptomatic therapy or no therapy, or from symptomatic therapy to 
no therapy. These results are essentially consistent with those reported by Kindelan-Calvo et al. [16] in their review. Wallasch et al. [26], evaluating a multidisciplinary intervention specific for headache, reported also better results: an attacks frequency reduction of $62.7 \%$, a disability degree reduction of 47.4 and an analgesic use reduction of 74.5\%. The improvement was unrelated to headache sub-types. Noteworthy, stress triggers attacks both in migraine and tension-type headache [29], even though their different pathophysiology. So, we can hypothesize that the influence of our intervention is on the amelioration of the negative influence of stress in triggering headache attacks.

Another point deserving attention is related to the good results showed by patients with chronic headache (about $40 \%$ of the initial sample) and the significant reduction of the rate of prophylaxis among these patients after the psychological treatment (from 38\% to $12 \%$ ). The interruption of prophylaxis was decided by neurologists after the end of the intervention. We stress the importance of non-drug treatment for patients with chronic headache, in consideration of the risks of drug overuse, the worst impact on quality of life and psychopathological milieu of this kind of patients compared to episodic headache.

Headache is a complex disabling condition, where biological factors interface with psychological ones. This is the reason why drug therapy may not be enough to ameliorate the burden of headache, and multidisciplinary interventions may allow dealing with the disorder from different points of view.

The proposed intervention integrates neurological/pharmacological intervention with a multimodal psycho-emotional approach.

Patients evaluated very positively the intervention: most of them considered it useful, consistent with expectations, and recommended it for persons suffering from headache.

Although almost all patients at the end of each meeting declared to feel an immediate benefit from relaxation, only few of them followed the recommendation of daily perform relaxation exercises at home. Since the explanation given was the difficulty with the autonomous management of relaxation, has been given to participants a recording of a relaxation session at the end of the treatment, in order to provide a support to their home practice.

Despite the evident limitations of the study (small sample size and lack of control group), the results are encouraging: not having side effects like drugs, psychoeducational intervention should be considered a useful instrument for both patients and physicians in the battle against headache. Further directions should address the adoption of a randomized, double-blind design to test the efficacy of such intervention. Another point that should be addressed in further studies is tailoring non-drug intervention according both to the psychological and/or psychopathological characteristics of the patients and headache characteristics. For example, alexithymia (a personality trait characterised by a reduced symbolic thought, restricted fantasy life, externally oriented cognitive thinking, difficulty in distinguishing feelings from bodily sensations and inadequacy in intuition and empathy), that has been recently associated with the occurrence of headache [30], could be a discriminant factor for proposing a patient for a cognitive-behavioral therapy. Moreover, the sub-type of headache may be a discriminant factor for the choice of the best non-drug therapy (no efficacy of cognitive-behavioral therapy has been recently evidenced in chronic post-traumatic headache [31]. Studies are warranted on this topic.
Education and self-management are important to all patients with headache and therefore an important part of the treatment which can be done by psychologists. This includes lifestyle education, selfmanagement, handling medication and risks of medication overuse. Even though detailed scientific data are sparse, psychologists are considered important members of multidisciplinary teams [17]. Nonpharmacological treatments are acknowledged as preventive methods especially for migraine according to neurological guidelines [32]. Furthermore, this kind of intervention has the advantage of reducing costs in the management of patients because it is a group intervention. Further studies are warranted to analyze the efficacy of such kind of intervention over time, comparing the efficacy of individual and group interventions as well.

In synthesis, we suggest to address headache reduction by a multidisciplinary work. Mixed psychoeducational group sessions and relaxation training may be highly efficacious in reducing the burden of headache. Studies are warranted in order to scrutinize which patient is best candidate for which therapy, integrating the drug and non-drug treatment.

\section{References}

1. Steiner TJ, Stovner LJ, Birbeck GL (2013) Migraine: the seventh disabler. J Headache Pain 53: 227-229.

2. Vos T, Flaxman AD, Naghavi M, Lozano R, Michaud C, et al. (2012) Years lived with disability (YLDs) for 1160 sequelae of 289 diseases and injuries 1990-2010: a systematic analysis for the Global Burden of Disease Study 2010. Lancet 380: 2163-2196.

3. Linde M, Gustavsson A, Stovner LJ, Steiner TJ, Barré J, et al. (2012) The cost of headache disorders in Europe: The Eurolight project. Eur J Neurol 19: 703-711.

4. Stovner Lj, Hagen K, Jensen R, Katsarava Z, Lipton R, et al. (2007) The global burden of headache: A documentation of headache prevalence and disability worldwide. Cephalalgia 27: 193-210.

5. Steiner TJ, Stovner LJ, Katsarava Z, Lainez JM, Lampl C, et al. (2014) The impact of headache in Europe: Principal results of the Eurolight project. J Headache Pain 21: 15-31.

6. Torelli P, Abrignani G, Berzieri L, Castellini P, Ferrante T, et al. (2010) Population-based pace study: Lifetime and past-year prevalence of headache in adults. Neurol Sci 31: 145-147.

7. Guidetti V, Galli F, Sheftell F (2010) Headache attributed to psychiatric disorders. Handb Clin Neurol 97: 657-662.

8. Sances G, Galli F, Ghiotto N, Allena M, Guaschino E, et al. (2013) Factors associated with a negative outcome of medication overuse headache - a three-year follow-up (the "care" protocol). Cephalalgia 33: 431-443.

9. Antonaci F, Voiticovschi-Iosob C, Di Stefano AL, Galli F, Ozge A, et al (2014) The evolution of headache from childhood to adulthood: A review of the literature. J Headache Pain 15: 15.

10. Gerber WD, Schoenen J (1998) Biobehavioral correlates in migraine: the role of hypersensitivity and information-processing dysfunction. Cephalalgia 18 Suppl 21: 5-11.

11. Andrasik F, Buse DC, Grazzi L (2009) Behavioral medicine for migraine and medication overuse headache. Curr Pain Headache Rep 13: 241-248.

12. Grazzi L, Bussone G (2011) What future for treatment of chronic migraine with medication overuse? Neurol Sci 32 Suppl 1: S19-22.

13. Schramm SH, Moebus S, Lehmann N, Galli U, Obermann M, et al. (2015) The association between stress and headache: A longitudinal populationbased study. Cephalalgia 35: 853-863.

14. Andrasik F (2004) Behavioral treatment of migraine: Current status and future directions. Expert Rev Neurother 4: 403-413.

15. Stanos S (2012) Focused review of interdisciplinary pain rehabilitation programs for chronic pain management. Curr Pain Headache Rep 16: 147-152. 
Citation: Andrighi E, Galli F, Massetto N, Strada I, Magno S, et al. (2016) Psychological Treatment for Headache: A Pilot Study on the Efficacy of Joint Psychoeducational Group and Relaxation Training. J Neurol Neurophysiol 7: 379. doi:10.4172/2155-9562.1000379

Page 5 of 5

16. Kindelan-Calvo P, Gil-Martinez A, Paris-Alemany A, Pardo-Montero J, Munoz-Garcia D, et al. (2014) Effectiveness of therapeutic patient education for adults with migraine. A systematic review and metaanalysis of randomized controlled trials. Pain Med 15: 1619-1636.

17. Gaul C, Visscher CM, Bhola R, Sorbi MJ, Galli F, et al. (2011) Team players against headache: Multidisciplinary treatment of primary headaches and medication overuse headache. J Headache Pain 12: 511-519.

18. Wahbeh H, Elsas SM, Oken BS (2008) Mind-body interventions: Applications in neurology. Neurology 70: 2321-2328.

19. Diamond S (2015) Psychological Approaches to Headache. Headache and Migraine Biology and Management. (1stedn), Academic Press, Elsevier, Chicago, IL, United States pp 239-251.

20. Sarkadi A, Rosenqvist U (2004) Experience-based group education in Type 2 diabetes: A randomised controlled trial. Patient Educ Couns 53 291-298.

21. Rachmani R, Slavacheski I, Berla M, Frommer-Shapira R, Ravid M (2005) Treatment of high-risk patients with diabetes: motivation and teaching intervention: A randomized, prospective 8-year follow-up study. J Am Soc Nephrol 16: S22-S26.

22. Louw A, Diener I, Butler DS, Puentedura EJ (2011) The effect of neuroscience education on pain, disability, anxiety, and stress in chronic musculoskeletal pain. Arch Phys Med Rehabil 92: 2041-2056.

23. Daviet JC, Bonan I, Caire JM, Colle F, Damamme L, et al. (2012) Therapeutic patient education for stroke survivors: Non-pharmacological management. A literature review. Ann Phys Rehabil Med 55: 641-656.
24. WHO Working Group (1998) Therapeutic Patient Education, Rev. 2 World Health Organization, Regional Office for Europe, Copenhagen.

25. Reed K (2010) Therapeutic Patient Education: Royal Prince Alfred Hospital, Sydney, Australia.

26. Wallasch TM, Angeli A, Kropp P (2012) Outcomes of a headache-specific cross-sectional multidisciplinary treatment program. Headache 52: 1094-1105.

27. (2013) Headache classification committee of the international headache society. The International Classification of Headache Disorders, 3rd edition (beta version). Cephalalgia 33: 629-808.

28. Andrasik F, Lipchik GL, McCrory DC, Wittrock DA (2005) Outcome measurement in behavioral headache research: Headache parameters and psychosocial outcomes. Headache 45: 429-437.

29. Martin PR (2016) Stress and Primary Headache: Review of the Research and Clinical Management. Curr Pain Headache Rep 20: 45.

30. Galli F, Caputi M, Sances G, Vegni E, et al. (2016) Alexithymia in chronic and episodic migraine: A comparative study. J Ment Health .

31. Kjeldgaard D, Forchhammer HB, Teasdale TW, Jensen RH (2014) Cognitive behavioural treatment for the chronic post-traumatic headache patient: A randomized controlled trial. The Journal of Headache and Pain 2: 81 .

32. Antonaci F, Dumitrache C, De Cillis I, Allena M (2010) A review of current European treatment guidelines for migraine. J Headache Pain 11: 13-19. 